\title{
METRICAL PROPERTIES OF BEST APPROXIMANTS
}

\author{
JOS BLOM
}

(Received 8 August 1990)

Communicated by J. H. Loxton

\begin{abstract}
A rational number is called a best approximant of the irrational number $\xi$ if it lies closer to $\xi$ than all rational numbers with a smaller denominator. Metrical properties of these best approximants are studied. The main tool is the two-dimensional ergodic system, underlying the continued fraction expansion.
\end{abstract}

1991 Mathematics subject classification (Amer. Math. Soc.): 11 J 70, 11 K 50.

\section{Introduction}

Let $x$ denote a real irrational number and let

$$
\left[B_{0} ; B_{1}, B_{2}, \ldots\right]
$$

be its regular continued fraction expansion. Denote the corresponding sequence of convergents by

$$
\left\{\frac{P_{n}}{Q_{n}}\right\}_{n=0}^{\infty}
$$

These convergents are defined by

$$
\frac{P_{n}}{Q_{n}}:=\left[B_{0} ; B_{1}, B_{2}, \ldots, B_{n}\right], \quad n \geq 0 .
$$

The approximation constant $\Theta(p / q, x)$ of a fraction $p / q$ with respect to $x$

(C) 1992 Australian Mathematical Society $0263-6115 / 92 \$ A 2.00+0.00$ 
is defined by the relation

$$
\left|x-\frac{p}{q}\right|=\frac{\Theta\left(\frac{p}{q}, x\right)}{q^{2}} .
$$

We shall write for abbreviation

$$
\boldsymbol{\Theta}_{n}=\boldsymbol{\Theta}_{n}(x)=\boldsymbol{\Theta}\left(\frac{P_{n}}{Q_{n}}, x\right), \quad n \geq 0 .
$$

The convergents provide very good rational approximations to $x$. This is expressed more precisely by the fact that the inequality $\Theta_{n}<1$ always holds. One also has the following theorem which was conjectured by $\mathrm{H}$. W. Lenstra and proved in [3]; see also [5] and [6].

(1.4) Theorem. For almost all $x$ the limiting distribution of the sequence $\left\{\boldsymbol{\Theta}_{n}\right\}_{n=0}^{\infty}$ exists and is equal to $(1 / \log 2) f$ where

$$
f(z)= \begin{cases}0 & \text { if } z \leq 0 \\ z & \text { if } 0 \leq z \leq \frac{1}{2} \\ 1-z+\log (2 z) & \text { if } \frac{1}{2} \leq z \leq 1 \\ \log 2 & \text { if } 1 \leq z\end{cases}
$$

Further, P. Lévy has shown a result about the denominators of the convergents, which says that for almost all $x$

$$
\lim _{n \rightarrow \infty} \frac{1}{n} \log Q_{n}=\frac{\pi^{2}}{12 \log 2}
$$

see [1].

(1.6) Definition. Let $D / E$ be a fraction in its lowest terms with positive denominator. We call $D / E$ a best approximant of $x$ if

$$
\left|\frac{p}{q}-x\right| \leq\left|\frac{D}{E}-x\right| \Rightarrow q>E
$$

holds for every rational number $p / q \neq D / E$.

Since $\mathbb{Q}$ is dense in $\mathbb{R}$ there are infinitely many best approximants of $x$. Ordering and enumerating them by increasing denominator we obtain the sequence of best approximants belonging to $x$. We denote this sequence by

$$
\left\{\frac{D_{k}}{E_{k}}\right\}_{k=1}^{\infty} \text {. }
$$


The question arises whether analogues of Theorem (1.4) and of Lévy's result exist for the best approximants. As we will see later, the answer is yes: for almost all $x$ and for all $z$

$$
\frac{1}{k} \#\left\{j \leq k: \boldsymbol{\theta}\left(\frac{D_{j}}{E_{j}}, x\right) \leq z\right\} \rightarrow 0 \text { if } k \rightarrow \infty
$$

and

$$
\frac{1}{k} \log E_{k} \rightarrow 0 \text { if } k \rightarrow \infty \text {; }
$$

see Corollary (2.17) and Corollary (2.19). These results, however, give too little information about the distribution of the approximation constants of the best approximants respectively the growth of the denominators $E_{k}$. In order to obtain more refined results, the set of best approximants is replaced by $S^{[C]}$, with $C$ an arbitrary positive constant, where

$$
\begin{array}{r}
S^{[C]}=S^{[C]}(x):=\left\{\frac{D}{E} \in \mathbb{Q}: \frac{D}{E} \text { is a best approximant of } x\right. \\
\text { and } \left.\theta\left(\frac{D}{E}, x\right) \leq C\right\} .
\end{array}
$$

We enumerate the elements of $S^{[C]}$ by increasing denominator and we denote the sequence thus obtained by

$$
\left\{\frac{D_{k}^{[C]}}{E_{k}^{[C]}}\right\}_{k=1}^{\# S^{[C]}}
$$

In Section 2 we will see that $\# S^{[C]}=\infty$ for almost all $x$ and we will prove more interesting analogues of Theorem (1.4) and Lévy's result for the elements of $S^{[C]}$.

(1.7) Remark. Many quantities in this article are dependent on $x$. However, for the convenience of the exposition we will not make visible this dependence in the notation of these quantities. Further we assume that the fractions $P / Q, L / M$ and $D / E$ are in their lowest terms and that their denominators are always positive.

Finally we point out that if we write "for almost all $x$ ", this is meant to be in the Lebesgue sense.

\section{Detailed study of the best approximants}

(2.1) Definition. Let $P_{-1}:=1, Q_{-1}:=0$ and let $P_{n}$ and $Q_{n}$ be as in (1.1) for $n \geq 0$. We call a rational number a mediant of $x$ if it is of the 
form

$$
\frac{L_{n}^{(B)}}{M_{n}^{(B)}}=\frac{B P_{n-1}+P_{n-2}}{B Q_{n-1}+Q_{n-2}},
$$

where $n$ and $B$ are integers with $n \geq 1$ and $0<B<B_{n}$.

Write

$$
\boldsymbol{\Theta}_{n}^{(B)}=\boldsymbol{\Theta}_{n}^{(B)}(x):=\boldsymbol{\Theta}\left(\frac{L_{n}^{(B)}}{M_{n}^{(B)}}, x\right), \quad n \geq 1,0<B<B_{n} .
$$

The best approximants are directly connected with the convergents and the mediants. The following two theorems describe exactly which fractions are best approximants in terms of convergents and mediants. The proofs of these two theorems can be found in [8], zweites Kapitel, Satz 18, 21 and 22.

(2.3) THEOREM. Every best approximant of $x$ is a convergent or a mediant of $x$.

(2.4) Theorem. (a) For $n \geq 1$ every convergent $P_{n} / Q_{n}$ is a best approximant; the convergent $P_{0} / Q_{0}$ is a best approximant if and only if $B_{1} \geq 2$.

(b) $A$ mediant $L_{n}^{(B)} / M_{n}^{(B)}$ is a best approximant if and only if $2 B>B_{n}$ or both $2 B=B_{n}$ and

$$
\left[B_{n} ; B_{n-1}, B_{n-2}, \ldots, B_{1}\right]>\left[B_{n} ; B_{n+1}, B_{n+2}, \ldots\right] .
$$

It is a consequence of Theorem (1.4) and Theorem 2.4(a) that for almost all $x$ the set $S^{[C]}$ contains infinitely many elements for all $C>0$.

Now we will do some preparatory work for Theorem (2.16) and Theorem (2.18). We note that we will use methods from [2] which in their turn go back to [3].

(2.5) Definition. Define the sequence $\left\{\left(T_{n}, V_{n}\right)\right\}_{n=0}^{\infty}$ by

$$
T_{n}=T_{n}(x):=\left[0 ; B_{n+1}, B_{n+2}, \ldots\right], \quad n \geq 0,
$$

and

$$
V_{n}=V_{n}(x):=\left[0 ; B_{n}, B_{n-1}, \ldots, B_{1}\right], \quad n \geq 0 .
$$

All the metrical results we will find are based on ergodic theory by using the following theorem.

(2.6) THEOREM. For every $x$ and every $n$ the pair $\left(T_{n}, V_{n}\right)$ is an element of the unit square $[0,1] \times[0,1]$. Moreover, for almost all $x$ the sequence $\left\{\left(T_{n}, V_{n}\right)\right\}_{n=0}^{\infty}$ is distributed over the unit square according to the density function

$$
\frac{1}{\log 2} \frac{1}{(1+T V)^{2}}
$$


The first part of the theorem is trivial. The second part is a corollary of a fundamental ergodic theorem of Ito, Nakada and Tanaka. For that fundamental theorem the reader is referred to [7] and for the corollary to [5].

(2.7) Theorem. Let $A(n, B, z, x)$ be defined by

$A(n, B, z, x):=\#\left\{j \leq n: \frac{L_{j}^{(B)}}{M_{j}^{(B)}}\right.$ exists, $\frac{L_{j}^{(B)}}{M_{j}^{(B)}}$ is a best

$$
\text { approximant of } \left.x \text { and } \boldsymbol{\theta}_{j}^{(B)} \leq z\right\} \text {. }
$$

Then for almost all $x$ and for all integers $B \geq 1$ the limit

$$
\lim _{n \rightarrow \infty} \frac{1}{n} A(n, B, z, x)
$$

exists and equals $(1 / \log 2) g^{(B)}$ where

$$
g^{(1)}(z)= \begin{cases}0 & \text { if } z \leq \frac{1}{2}, \\ -\frac{1}{2}+z-\frac{1}{2} \log (2 z) & \text { if } \frac{1}{2} \leq z \leq \frac{2}{3}, \\ \frac{1}{2}-\frac{1}{2} z+\frac{1}{2} \log \left(\frac{9}{8} z\right) & \text { if } \frac{2}{3} \leq z \leq 1, \\ \frac{1}{2} \log \frac{9}{8} & \text { if } 1 \leq z,\end{cases}
$$

and where for integers $B \geq 2$

$$
g^{(B)}(z)= \begin{cases}g_{0}^{(B)}(z)=0 & \text { if } z \leq \frac{B}{B+1}, \\ g_{1}^{(B)}(z)=-1+\frac{B+1}{B} z-\log \left(\frac{B+1}{B} z\right) & \text { if } \frac{B}{B+1} \leq z \leq \frac{B+1}{B+2} \\ g_{2}^{(B)}(z)=\frac{1}{B(B+1)} z+\log \frac{B(B+2)}{(B+1)^{2}} & \text { if } \frac{B+1}{B+2} \leq z \leq \frac{B}{2}, \\ g_{3}^{(B)}(z)=\frac{1}{2}-\frac{1}{B+1} z+\frac{1}{2} \log \left(\frac{2 B(B+2)^{2}}{(B+1)^{4}} z\right) & \text { if } \frac{B}{2} \leq z \leq \frac{B+1}{2}, \\ g_{4}^{(B)}(z)=\frac{1}{2} \log \frac{B(B+2)^{2}}{(B+1)^{3}} & \text { if } \frac{B+1}{2} \leq z .\end{cases}
$$


Proof. If we define

$$
\begin{aligned}
\mathscr{A}^{(B)}:= & \left\{(T, V): \frac{1}{2 B}<T \leq \frac{1}{B+1}, 0 \leq V \leq 1\right\} \\
& \cup\left\{(T, V): \frac{1}{2 B+1}<T \leq \frac{1}{2 B}, V>\frac{1}{T}-2 B\right\},
\end{aligned}
$$

it follows from Theorem (2.3) and Theorem (2.4) that $\left(T_{n-1}, V_{n-1}\right) \in \mathscr{A}^{(B)}$ if and only if $L_{n}^{(B)} / M_{n}^{(B)}$ exists and is a best approximant of $x$. From (1.3) and (2.2), from Definitions (2.1) and (2.5) and from the basic relations

$$
\Theta_{n}=\frac{T_{n}}{1+T_{n} V_{n}} \text { and } \Theta_{n-1}=\frac{V_{n}}{1+T_{n} V_{n}}
$$

using the alternating way in which the sequence of convergents approaches $x$, we can derive that

$$
\boldsymbol{\Theta}_{n}^{(B)}=\frac{\left(1-B T_{n-1}\right)\left(B+V_{n-1}\right)}{1+T_{n-1} V_{n-1}}, \quad n \geq 1,0<B<B_{n} .
$$

Defining

$$
\mathscr{H}^{(B)}(z):=\left\{(T, V):(1-(B+z) T) V \leq B^{2} T+z-B\right\} \subset \mathbb{R} \times \mathbb{R},
$$

we can conclude that

$$
\left(T_{n-1}, V_{n-1}\right) \in \mathscr{H}^{(B)}(z) \Leftrightarrow \Theta_{n}^{(B)} \leq z, \quad\left(T_{n-1}, V_{n-1}\right) \in \mathscr{A}^{(B)} .
$$

So together with Theorem (2.6) we obtain for almost all $x$

$$
\begin{aligned}
\lim _{n \rightarrow \infty} & \frac{1}{n} A(n, B, z, x)=\frac{1}{\log 2} \iint_{\mathscr{A}^{(B)} \cap \mathscr{E}^{(B)}(z)} \frac{d V d T}{(1+T V)^{2}} \\
= & \frac{1}{\log 2} g^{(B)}(z) .
\end{aligned}
$$

Now we have to compute this double integral. By the definition of $\mathscr{H}^{(B)}(z)$, we see that $\mathscr{H}^{(B)}(z)$ is the area between or on the two branches of the hyperbola

$$
V=\frac{B^{2} T+z-B}{1-(B+z) T}
$$

With increasing $z$, the hyperbola moves to the left; the left-hand branch of the hyperbola moves through $\mathscr{A}^{(B)}$ while the right-hand branch intersects $\mathscr{A}^{(B)}$ for no value of $z$. The following figure gives schematically the possible positions of the left-hand branch of the hyperbola (indicated by a dotted line) with respect to $\mathscr{A}^{(B)}$. 


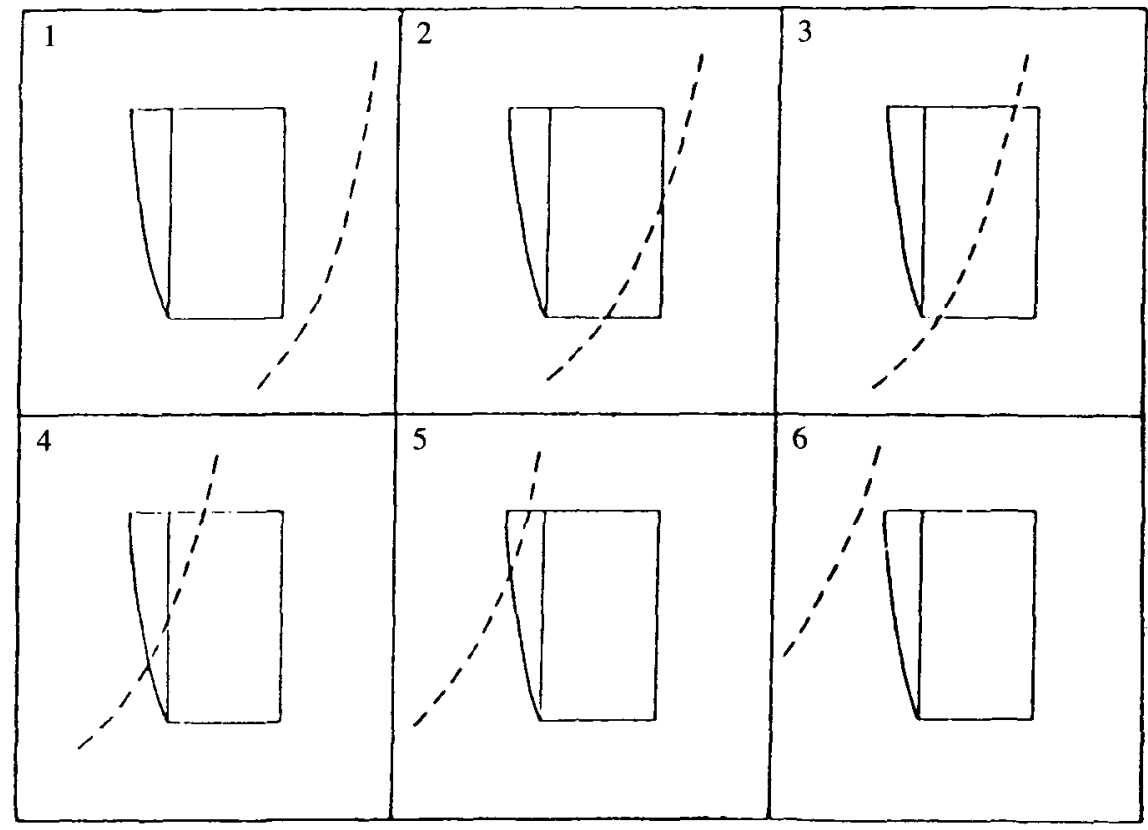

FIGURE 1.

With the help of Figure 1 we find, after some computations, for $B \geq 2$, that

$$
\begin{aligned}
& z<\frac{B}{B+1} \Rightarrow \mathscr{A}^{(B)} \cap \mathscr{H}^{(B)}(z)=\varnothing, \\
& \frac{B}{B+1} \leq z \leq \frac{B+1}{B+2} \Rightarrow \mathscr{A}^{(B)} \cap \mathscr{H}^{(B)}(z) \\
& \quad=\left\{(T, V): \frac{B-z}{B^{2}} \leq T \leq \frac{1}{B+1}, 0 \leq V \leq \frac{B^{2} T+z-B}{1-(B+z) T}\right\}, \\
& \frac{B+1}{B+2} \leq z<\frac{B}{2} \Rightarrow \mathscr{A}^{(B)} \cap \mathscr{H}^{(B)}(z) \\
& \quad=\left\{(T, V): \frac{B-z}{B^{2}} \leq T \leq \frac{B+1-z}{B^{2}+B+z}, 0 \leq V \leq \frac{B^{2} T+z-B}{1-(B+z) T}\right\} \\
& \cup\left\{(T, V): \frac{B+1-z}{B^{2}+B+z}<T \leq \frac{1}{B+1}, 0 \leq V \leq 1\right\},
\end{aligned}
$$




$$
\begin{aligned}
& \frac{B}{2} \leq z \leq \frac{B+(B+1)}{2 B+1} \Rightarrow \mathscr{A}^{(B)} \cap \mathscr{H}^{(B)}(z) \\
& =\left\{(T, V): \frac{1}{B+2 z}<T \leq \frac{1}{2 B}, \frac{1}{T}-2 B<V \leq \frac{B^{2} T+z-B}{1-(B+z) T}\right\} \\
& \cup\left\{(T, V): \frac{1}{2 B}<T \leq \frac{B+1-z}{B^{2}+B+z}, 0 \leq V \leq \frac{B^{2} T+z-B}{1-(B+z) T}\right\} \\
& \cup\left\{(T, V): \frac{B+1-z}{B^{2}+B+z}<T \leq \frac{1}{B+1}, 0 \leq V \leq 1\right\}, \\
& \frac{B(B+1)}{2 B+1} \leq z \leq \frac{B+1}{2} \Rightarrow \mathscr{A}^{(B)} \cap \mathscr{H}^{(B)}(z) \\
& =\left\{(T, V): \frac{1}{B+2 z}<T \leq \frac{B+1-z}{B^{2}+B+z}, \frac{1}{T}-2 B<V \leq \frac{B^{2} T+z-B}{1-(B+z) T}\right\} \\
& \cup\left\{(T, V): \frac{B+1-z}{B^{2}+B+z}<T \leq \frac{1}{2 B}, \frac{1}{T}-2 B<V \leq 1\right\} \\
& \cup\left\{(T, V): \frac{1}{2 B}<T \leq \frac{1}{B+1}, 0 \leq V \leq 1\right\} \text {, } \\
& z \geq \frac{B+1}{2} \Rightarrow \mathscr{A}^{(B)} \cap \mathscr{H}^{(B)}(z) \\
& =\left\{(T, V): \frac{1}{2 B+1}<T \leq \frac{1}{2 B}, \frac{1}{T}-2 B<V \leq 1\right\} \\
& \cup\left\{(T, V): \frac{1}{2 B}<T \leq \frac{1}{B+1}, 0 \leq V \leq 1\right\} .
\end{aligned}
$$

The case $B=1$ has to be considered separately, because $\mathscr{A}^{(1)}$ has a different form. We leave this case to the reader, as it presents no further difficulties.

Now the double integral in $(2.8)$ can be evaluated by irksome but straightforward calculations and so one finds the expressions for $g^{(B)}(z)$ from the theorem. This is also left to the reader. Remark that the case $B / 2 \leq z \leq$ $B(B+1) /(2 B+1)$ and the case $B(B+1) /(2 B+1) \leq z \leq(B+1) / 2$ yield the same expression for $g^{(B)}(z)$, as is to be expected when one compares the cases 4 and 5 from Figure 1 .

We immediately proceed with the following theorem.

(2.9) ThEOREM. Let $g^{(B)}$ be as in Theorem (2.7) and define $h$ by

$$
h(z):=\sum_{B=1}^{\infty} g^{(B)}(z)
$$


Then

$$
h(z)= \begin{cases}0 & \text { if } z \leq \frac{1}{2}, \\ -\frac{1}{2}+z-\frac{1}{2} \log (2 z) & \text { if } \frac{1}{2} \leq z \leq 1, \\ \frac{1}{2}+\frac{1}{2} \log \left(\frac{1}{2} z\right) & \text { if } 1 \leq z .\end{cases}
$$

For the graph of the function $h$, see Figure 2 .

Proof. For $z<2 / 3$ the theorem is easily verified. Suppose that $2 / 3 \leq$ $z<1$. We define the integer $k$ by $k /(k+1) \leq z<(k+1) /(k+2)$. Then $k \geq 2$ and we get

$$
h(z)=g^{(1)}(z)+\sum_{B=2}^{k-1} g_{2}^{(B)}(z)+g_{1}^{(k)}(z)+\sum_{B=k+1}^{\infty} g_{0}^{(B)}(z)=-\frac{1}{2}+z-\frac{1}{2} \log (2 z) \text {. }
$$

For $z \geq 1$ let the integer $k$ be determined by $k / 2 \leq z<(k+1) / 2$. Then $k \geq 2$ and we find

$$
h(z)=g^{(1)}(z)+\sum_{B=2}^{k-1} g_{4}^{(B)}(z)+g_{3}^{(k)}(z)+\sum_{B=k+1}^{\infty} g_{2}^{(B)}(z)=\frac{1}{2}+\frac{1}{2} \log \left(\frac{1}{2} z\right) .
$$

(2.10) Definition. For all $C>0$ and for all integers $n \geq 0$ we define $k_{n}^{[C]}=k_{n}^{[C]}(x)$ by

$$
k_{n}^{[C]}:=\#\left\{k \in \mathbb{N}: E_{k} \leq Q_{n} \text { and } \boldsymbol{\theta}\left(\frac{D_{k}}{E_{k}}, x\right) \leq C\right\}
$$

(2.11) REMARK. $\left\{E_{k}^{[C]}\right\}_{k=1}^{\# S^{[C]}}$ is an increasing sequence, so we have

$$
\left\{E_{k} \leq Q_{n}: \boldsymbol{\theta}\left(\frac{D_{k}}{E_{k}}, x\right) \leq C\right\}=\left\{E_{k}^{[C]}: k=1,2, \ldots, k_{n}^{[C]}\right\} .
$$

Therefore, if $k_{n}^{[C]} \neq 0$ and $k_{n}^{[C]}+1 \leq \# S^{[C]}$ then

$$
E_{k_{n}^{[C]}}^{[C]} \leq Q_{n}<E_{k_{n}^{[C]}+1}^{[C]}
$$

Now we have the tools to prove the following important theorem.

(2.12) TheOREM. For almost all $x$

$$
\lim _{n \rightarrow \infty} \frac{k_{n}^{[C]}}{n}=\frac{1}{\log 2}(f(C)+h(C))= \begin{cases}\frac{1}{\log 2} C & \text { if } 0<C \leq \frac{1}{2}, \\ \frac{1}{2 \log 2}(1+\log (2 C)) & \text { if } \frac{1}{2} \leq C\end{cases}
$$




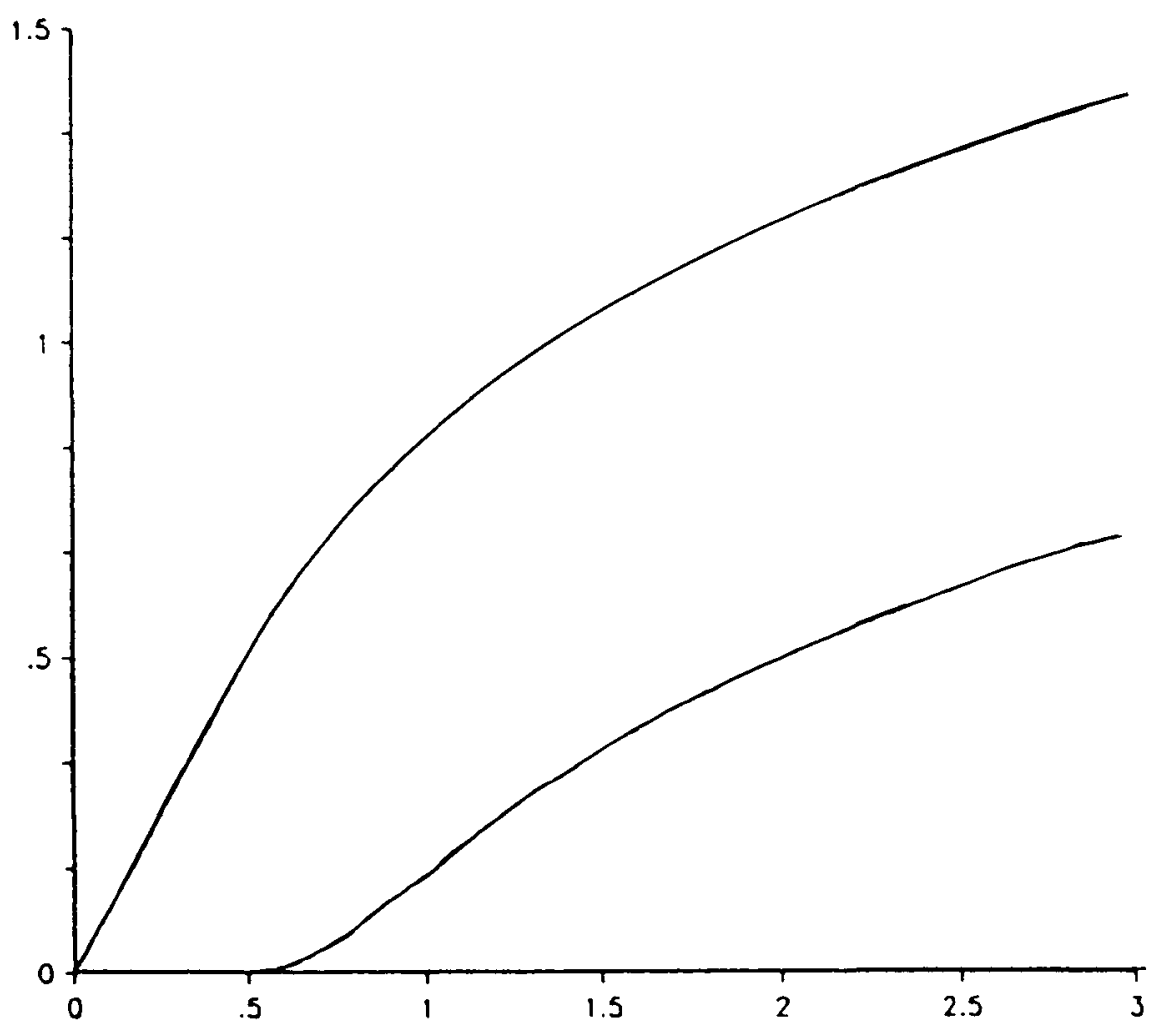

Figure 2. Graphs of the functions $h$ and $f+h$

for all $C>0$, with the $f$ from Theorem (1.4) and the $h$ from Theorem (2.9).

Proof. We take a value of $x$ for which the sequence $\left\{\left(T_{n}, V_{n}\right)\right\}_{n=0}^{\infty}$ is distributed according to the density function denoted in Theorem (2.6). This implies that for this $x$ Theorem (1.4) and Theorem (2.7) hold (see the proofs of these theorems). Let $C$ be an arbitrary positive constant. Since

$$
Q_{n-1}<M_{n}^{(1)}<M_{n}^{(2)}<\cdots<M_{n}^{\left(B_{n}-1\right)}<Q_{n}, \quad n \geq 2,
$$

we get for all integers $b \geq 1$ :

$$
\begin{aligned}
\liminf _{n \rightarrow \infty} \frac{k_{n}^{[C]}}{n} & \geq \liminf _{n \rightarrow \infty} \frac{1}{n} \#\left\{j \leq n: \Theta_{j} \leq C\right\}+\liminf _{n \rightarrow \infty} \sum_{B=1}^{b} \frac{1}{n} A(n, B, z, x) \\
& =\frac{1}{\log 2}\left(f(C)+\sum_{B=1}^{b} g^{(B)}(C)\right)
\end{aligned}
$$


and when we let $b \rightarrow \infty$ we find

$$
\liminf _{n \rightarrow \infty} \frac{k_{n}^{[C]}}{n} \geq \frac{1}{\log 2}(f(C)+h(C))
$$

The estimation of $\limsup _{n \rightarrow \infty} k_{n}^{[C]} / n$ is somewhat more complicated. For all integers $b \geq 1$ one has

$$
\begin{aligned}
\limsup _{n \rightarrow \infty} \frac{k_{n}^{[C]}}{n} \leq & \limsup _{n \rightarrow \infty} \frac{1}{n} \#\left\{j \leq n: \Theta_{j} \leq C\right\}+\limsup _{n \rightarrow \infty} \sum_{B=1}^{b-1} \frac{1}{n} A(n, B, z, x) \\
& +\limsup _{n \rightarrow \infty} \sum_{B=b}^{\infty} \frac{1}{n} \#\left\{j \leq n:\left(T_{j-1}, V_{j-1}\right) \in \mathscr{A}^{(B)} \cap \mathscr{X}^{(B)}(C)\right\} \\
\leq & \frac{1}{\log 2}\left(f(C)+\sum_{B=1}^{b-1} g^{(B)}(C)\right) \\
& +\limsup _{n \rightarrow \infty} \sum_{B=b}^{\infty} \frac{1}{n} \#\left\{j \leq n: \frac{B-C}{B^{2}} \leq T_{j-1} \leq \frac{1}{B+1}\right\} .
\end{aligned}
$$

It is easy to verify that

$$
\frac{B-C}{B^{2}} \geq \frac{1}{(B+C)+1}>\frac{1}{(B+C+1)+1}, \quad B \geq C^{2}+C .
$$

This implies that for all $a \in \mathbb{R}$

$$
\#\left\{B \geq C^{2}+C: a \in\left[\frac{B-C}{B^{2}}, \frac{1}{B+1}\right]\right\} \leq C+1 .
$$

So we can conclude that

$$
\begin{aligned}
\sum_{B=b}^{\infty} & \frac{1}{n} \#\left\{j \leq n: \frac{B-C}{B^{2}} \leq T_{j-1} \leq \frac{1}{B+1}\right\} \\
& \leq(C+1) \frac{1}{n} \#\left\{j \leq n: 0 \leq T_{j-1} \leq \frac{1}{b+1}\right\}, \quad b \geq C^{2}+C .
\end{aligned}
$$

When we combine (2.13) and (2.14) and let $b \rightarrow \infty$ this yields

$$
\limsup _{n \rightarrow \infty} \frac{k_{n}^{[C]}}{n} \leq \frac{1}{\log 2}(f(C)+h(C)) \text {. }
$$

Let us take an $x$ for which the limit relation of Theorem (2.12) holds and let $C>0$. We fix $k \geq k_{0}^{[C]}+1$ and we define $n=n(k, x)$ by

$$
k_{n}^{[C]}+1 \leq k \leq k_{n+1}^{[C]} \text {. }
$$


By Theorem (2.12) the existence and uniqueness of this $n$ is guaranteed. For $0<z \leq C$ we have

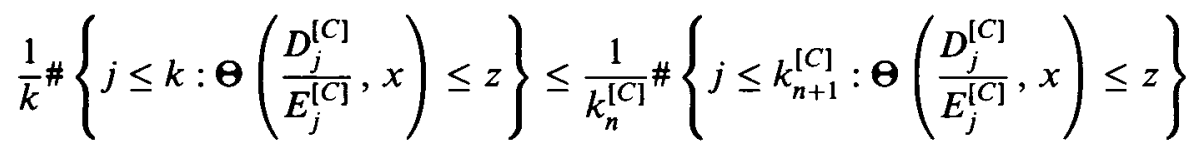

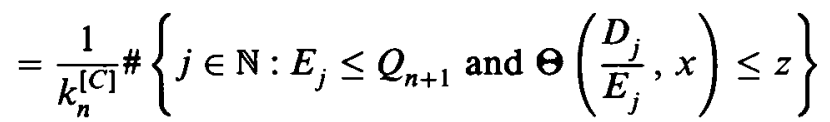

$$
\begin{aligned}
& =\frac{1}{k_{n}^{[C]}} k_{n+1}^{[z]}
\end{aligned}
$$

and similarly

$$
\frac{1}{k} \#\left\{j \leq k: \theta\left(\frac{D_{j}^{[C]}}{E_{j}^{[C]}}, x\right) \leq z\right\} \geq \frac{1}{k_{n+1}^{[C]}} k_{n}^{[z]} .
$$

When we now let $k \rightarrow \infty$ (and so $n \rightarrow \infty$ ), Theorem (2.12) yields the nontrivial part of the next theorem about the distribution of the approximation constants of the elements of $S^{[C]}$.

(2.16) Theorem. let $C \geq 0$. For almost all $x$ the limit

$$
\lim _{k \rightarrow \infty} \frac{1}{k} \#\left\{j \leq k: \Theta\left(\frac{D_{j}^{[C]}}{E_{j}^{[C]}}, x\right) \leq z\right\}
$$

exists and equals $h^{[C]}(z)$ where

$$
h^{[C]}(z)= \begin{cases}0 & \text { if } z \leq 0, \\ \frac{f(z)+h(z)}{f(C)+h(C)} & \text { if } 0 \leq z \leq C, \\ 1 & \text { if } C \leq z\end{cases}
$$

and

$$
\frac{f(z)+h(z)}{f(C)+h(C)}= \begin{cases}\frac{1}{C} z & \text { if } 0 \leq z \leq C, 0<C \leq \frac{1}{2}, \\ \frac{1}{1+\log (2 C)} 2 z & \text { if } 0 \leq z \leq \frac{1}{2}, \frac{1}{2} \leq C, \\ \frac{1}{1+\log (2 C)}(1+\log (2 z)) & \text { if } \frac{1}{2} \leq z \leq C, \frac{1}{2} \leq C .\end{cases}
$$

Since

$$
\left\{j \leq k: \boldsymbol{\Theta}\left(\frac{D_{j}}{E_{j}}, x\right) \leq z\right\} \subset\left\{j \leq k: \boldsymbol{\Theta}\left(\frac{D_{j}^{[C]}}{E_{j}^{[C]}}, x\right) \leq z\right\}, \quad z \leq C,
$$

we obtain the following corollary. 
(2.17) COROllary. For almost all $x$

$$
\lim _{k \rightarrow \infty} \frac{1}{k} \#\left\{j \leq k: \Theta\left(\frac{D_{j}}{E_{j}}, x\right) \leq z\right\}=0 .
$$

Now let $x$ have a value for which (1.5) as well as the limit relation of Theorem (2.12) hold. Again, let $C>0$ and let $k$ and $n$ be as in (2.15). Using Remark (2.11) we obtain

$$
\frac{1}{k_{n+1}^{[C]}} \log Q_{n}<\frac{1}{k} \log E_{k}^{[C]} \leq \frac{1}{k_{n}^{[C]}+1} \log Q_{n+1}
$$

and this, together with (1.5) and Theorem (2.12), yields an extended analogue of Lévy's result of the denominators for the best approximants.

(2.18) TheOREM. For almost all $x$

$$
\lim _{k \rightarrow \infty} \frac{1}{k} \log E_{k}^{[C]}=\frac{\pi^{2}}{12(f(C)+h(C))}= \begin{cases}\frac{\pi^{2}}{12 C} & \text { if } 0<C \leq \frac{1}{2}, \\ \frac{\pi^{2}}{6(1+\log (2 C))} & \text { if } \frac{1}{2} \leq C\end{cases}
$$

for all $C>0$.

(2.19) Corollary. For almost all $x$

$$
\lim _{k \rightarrow \infty} \frac{1}{k} \log E_{k}=0 \text {. }
$$

(2.20) Remark. It was proved by P. Erdös [4] (see also W. M. Schmidt [9]), that for almost all $x$

$$
\lim _{n \rightarrow \infty} \frac{U(x, z, n)}{\log n}=\frac{12}{\pi^{2}} z, \quad 0 \leq z,
$$

where

$$
U(x, z, n)=\#\{(p, q) \in \mathbb{Z} \times \mathbb{N}:(p, q)=1, \quad \Theta(p / q, x) \leq z, q \leq n\} .
$$

If we define

$$
\begin{array}{r}
U_{1}(x, z, n)=\#\{(p, q) \in \mathbb{Z} \times \mathbb{N}: \Theta(p / q, x) \leq z, q \leq n, \\
p / q \text { is a convergent of } x\}
\end{array}
$$

and

$$
\begin{array}{r}
U_{2}(x, z, n)=\#\{(p, q) \in \mathbb{Z} \times \mathbb{N}: \Theta(p / q, x) \leq z, q \leq n, \\
p / q \text { is a best approximant of } x\},
\end{array}
$$


then we find as a corollary of Theorems (1.4), (1.5) and (2.12) that for almost all $x$

$$
\lim _{n \rightarrow \infty} \frac{U_{1}(x, z, n)}{\log n}=\frac{12}{\pi^{2}} f(z)
$$

and

$$
\lim _{n \rightarrow \infty} \frac{U_{2}(x, z, n)}{\log n}=\frac{12}{\pi^{2}}(f(z)+h(z)),
$$

with the $f$ from Theorem (1.4) and the $h$ from Theorem (2.9).

\section{References}

[1] P. Billingsley, Ergodic theory and information (John Wiley, New York, 1965).

[2] W. Bosma, 'Approximation by mediants', Math. of Comp. 54 (1990), 421-434.

[3] W. Bosma, H. Jager and F. Wiedijk, 'Some metrical observations on the approximation by continued fractions', Indag. Math. 45 (1983), 281-299.

[4] P. Erdös, 'Some results on diophantine approximation', Acta Arithmetica 5 (1959), 359369.

[5] H. Jager, 'Continued fractions and ergodic theory', Transcendental Numbers and Related Topics, RIMS Kokyuroku 599, (Kyoto University, Kyoto, Japan 1986), pp. 55-59.

[6] D. E. Knuth, 'The distribution of continued fraction approximations', J. Number Theory 19 (1984), 443-448.

[7] H. Nakada, S. Ito and S. Tanaka, 'On the invariant measure for the transformations associated with some real continued fractions', Keio Engineering Reports 30 (1977), 159-175.

[8] O. Perron, Die Lehre von den Kettenbrüchen, Dritte verbesserte Auflage, (B. G. Teubner, Stuttgart 1954).

[9] W. M. Schmidt, 'A metrical theorem in Diophantine approximations', Canad. J. Math. 11 (1960), 619-631.

Harrelaers 11

1852 KT, Heiloo

the Netherlands 